\title{
When the worry is worse than the actual illness
}

Eric Jarmon, DO, Alison Sullivan, MD, Ulrick Vieux, DO, MS, and Christina Boyajian, LMHC

\section{How would you handle this case?}

Answer the challenge questions at MDedge.com/ psychiatry and see how your colleagues responded

\section{$f$}

Discuss this article at www.facebook.com/ MDedgePsychiatry

\section{HISTORY In pain and isolated}

Since being diagnosed with SMAS, Ms. S has had approximately 30 medical and 7 ED visits for SMAS-related pain. Ms. S was referred to the outpatient clinic for ongoing support and treatment for SMAS.

Because of her pain and anxiety, Ms. S, a junior in high school, no longer attends school but has been working with a tutor. Ms. S says that some of her loneliness and hopelessness are due to the social isolation of being tutored at home. She states that she has been "out of sight and out of mind" from her friends. She also reports feeling different from them due to the pain brought on by SMAS.

Ms. $S$ and her mother live in public housing. Ms. $S$ says that overall, she has a good relationship with her mother, but that in certain situations, her mother's anxiety causes her significant frustration and anxiety.

\section{EVALUATION Transient suicidal thoughts}

A physical examination reveals superficial lacerations to Ms. S's left arm. Although she appears thin, her current body mass index (BMI) is $20.4 \mathrm{~kg} / \mathrm{m}^{2}$, which is within normal range. She says she sees herself as "underweight" and "not fat at all." Ms. S reports that she likes food and enjoyed eating until it became too painful following her SMAS diagnosis. Ms. $\mathrm{S}$ denies a history of binging or

Dr. Jarmon is Attending Physician, Garnet Health Medical Center, Middletown, New York. Dr. Sullivan is Attending Physician, Garnet Health Medical Center, Middletown, New York. Dr. Vieux is Behavioral Health Chairperson and Director of Psychiatry Residency Program, Garnet Health Medical Center, Middletown, New York. Ms. Boyajian is a Licensed Mental Health Counselor, Garnet Health Medical Center, Middletown, New York.

Disclosures

The authors report no financial relationships with any companies whose products are mentioned in this article, or with manufacturers of competing products.

doi: $10.12788 /$ cp.0022 
purging. Results from her laboratory workup and all values are within normal limits.

During the initial interview, Ms. S's mother says they came to the ED because Ms. $S$ urgently needs a psychiatric evaluation so she can be cleared for gastrointestinal (Gl) surgery and placement of a nasogastric tube. Her mother says a surgeon from a different hospital told them that her insurance company required a psychiatric evaluation to rule out anorexia nervosa before they would authorize the Gl surgery. When asked why psychiatry at this hospital was not consulted, Ms. S's mother does not answer.

When asked about the symptoms she has been experiencing, Ms. $\mathrm{S}$ says that her sleep has been poor because of increased pain and excessive worrying about her health. She has limited her food intake. Ms. S reports that after eating, she lays on her left side to alleviate pain and help the food move through her body.

Ms. S says she feels anxious and depressed due to her SMAS diagnosis, her mother's online research and oversharing of poor prognoses, and being isolated from her friends. Most of her time outside the home is spent attending medical appointments with specialists. Several months ago, Ms. $\mathrm{S}$ had seen a psychotherapist, but her mother was unhappy with the treatment recommendations, which included seeking care from a nutritionist and joining group therapy. Ms. S's mother says she ended her daughter's psychotherapy because she was unable to obtain a signature ruling out anorexia nervosa within the first few appointments.

Ms. S also says she has had passive suicidal thoughts during the past month, usually twice a week. She reports that these thoughts lasted as long as several hours and were difficult to control, but she has no specific plan or intent. Ms. $S$ denies current suicidal thoughts or ideation, and works with the treatment team to complete a safety plan, which she signs. Other than her recent visit to the ED, Ms. S denies any other thoughts or behaviors of self-injury or suicide.
Table 1

\section{DSM-5 criteria for somatic symptom disorder}

Patient has $\geq 1$ somatic symptoms that cause enough distress so as to significantly disrupt daily functioning. The diagnosis also requires at least 1 of the following 3 criteria:

Thoughts about the seriousness of one's illness are either disproportionate or persistent

Anxiety is persistently high with regard to health or symptoms

An excessive amount of time or energy is devoted to these health concerns or symptoms

Source: Reference 1

Which of the following should be considered in the differential diagnosis?
a) major depressive disorder (MDD)
b) anorexia nervosa
c) somatic symptom disorder
d) factitious disorder imposed on self (FDIS)
e) factitious disorder imposed on another (FDIA)
f) all of the above

\section{The authors' observations}

The treatment team considered the following conditions as part of Ms. S's differential diagnosis:

Major depressive disorder. The team was able to rule out MDD because Ms. S's depression was attributed to SMAS. Ms. S reported that all depressive symptoms were manageable or nonexistent before the onset of pain from SMAS. There was no direct pathophysiological consequence of another medical condition. Ms. S was clear that her symptoms of anxiety and depression began after she was isolated from her friends and began having difficulty understanding her diagnosis and prognosis.

Anorexia nervosa also was ruled out. According to the DSM-5, a diagnosis of anorexia nervosa requires the following 3 criteria $^{1}$ :

\section{Clinical Point}

Patients with somatic symptom disorder often have $\geq 1$ somatic symptoms that cause enough distress to disrupt daily functioning 


\section{Clinical Point}

\section{Ms. S's pediatric surgeon and gastroenterologist both reported concerns of factitious disorder}

- restriction of food intake resulting in significantly low body weight (defined as weight that is less than "minimally normal") relative to age, gender, or development

- intense fear of gaining weight, or persistent behaviors that interfere with weight gain

- disturbance in the way in which one's body weight or shape is experienced, undue influence of body weight or shape on selfevaluation, or lack of insight with regard to seriousness of current low body weight.

Although Ms. S appeared thin, her BMI was within normal range. She added that she likes food and enjoyed eating, but that her medical condition made it too painful. Lastly, Ms. S denied a history of binging or purging.

Somatic symptom disorder. Table $\mathbf{1}^{1}$ (page 47) outlines the DSM-5 criteria for somatic symptom disorder. A diagnosis of somatic symptom disorder requires $\geq 1$ somatic symptoms that cause enough distress to significantly disrupt daily functioning. Furthermore, the patient is characteristically preoccupied with excessive thoughts, feelings, or behaviors as they relate to the health concern, manifested by at least 1 of the following: disproportionate and constant thoughts about the seriousness of the somatic symptom; continuous high anxiety with regard to health or somatic symptoms; and excessive time and energy spent worrying about these somatic symptoms or "health concerns."

Factitious disorder imposed on self. An individual with FDIS chronically stimulates, induces, or aggravates illnesses to gain the status of being a patient.

\section{Factitious disorder imposed on another}

is the deliberate feigning or production of symptoms in another individual who is under the perpetrator's supervision. ${ }^{1}$ Table $2^{3}$ (page 49) lists clinical indicators that raise suspicion for FDIA.

Before a diagnosis of somatic symptom disorder, FDIS, or FDIA could be established or ruled out, it was imperative to gather collateral information from other clinicians involved in Ms. S's care. Ms. S and her mother had sought out help from a pediatric surgeon, a pediatric gastroenterologist, a pediatrician, and a psychotherapist.

\section{EVALUATION Collateral information} After Ms. S's mother signs consent forms for exchange of information, the treatment team reaches out to the other clinicians. The therapist confirms that Ms. S's mother had ended her daughter's treatment after she was unable to quickly obtain documentation to rule out anorexia nervosa.

Both the pediatric surgeon and gastroenterologist report concerns of FDIA, which is why both clinicians had referred Ms. S and her mother to psychiatry. The pediatric surgeon states that on one occasion when he interviewed Ms. S separately from her mother, she seemed to be going down a checklist of symptoms. The surgeon reports that there was a partial occlusion of the superior mesenteric artery, confirming the diagnosis of SMAS, but he believed it was not severe enough to explain the symptoms Ms. S reported. The surgeon had scheduled another imaging appointment for 1 month later.

The pediatric gastroenterologist reports that Ms. S's mother had demanded surgery and nasogastric tube placement for her daughter, which raised suspicion of FDIA. The gastroenterologist had convinced Ms. S and her mother to start low-dose doxepin, $20 \mathrm{mg}$ twice a day, for anxiety, sleep, and abdominal pain.

Lastly, the pediatrician reports that she had not seen Ms. $S$ for several months but stated that Ms. $S$ always has been in the low normal BMI range. The pediatrician also reports that 6 months ago, the patient and her mother were frantically visiting EDs and scheduling doctor's appointments.

Based on this collateral information, what should be the next step?

a) remove Ms. S from her home 
b) continue doxepin

c) begin individual and/or family psychotherapy for Ms. S and her mother

d) explore placing Ms. S with another family member

\section{The authors' observations}

The treatment team decided that Ms. S was not in imminent danger, and felt it was important to keep her in treatment without raising her mother's suspicion. The team agreed to raise these concerns to the police, child protective services, and risk management if Ms. S's health suddenly deteriorated or if her mother decided to remove Ms. S from our care.

The treatment team at the outpatient psychiatry clinic agreed that Ms. S did not currently meet criteria for anorexia nervosa, MDD, FDIS, or FDIA. However, Ms. $S$ reported worries particular to persistent abdominal pain that was exacerbated by either eating or going to bed at night, which indicated that somatic symptom disorder was her likely diagnosis. Further, she endorsed a high level of anxiety and depression with regard to this somatic complaint that interfered with her daily activities and consumed an excessive amount of time, which also pointed to somatic symptom disorder. As a result of this diagnosis, the treatment team helped Ms. S manage her somatic symptoms and monitored for any other changes in her symptoms.

Generally, cognitive-behavioral therapy (CBT) and mindfulness-based therapy may help relieve symptoms associated with somatic symptom disorder. ${ }^{4}$

\section{TREATMENT Therapy sessions and medication management}

At the psychiatric clinic, Ms. S is scheduled for biweekly therapy sessions with a social worker and biweekly appointments with a senior psychiatry resident for medication

\section{Table 2}

\section{Clinical indicators that may suggest factitious disorder imposed by another}

\section{Symptoms are extremely unusual or physiologically unexplainable}

Repeated hospitalizations and workups by a series of clinicians do not offer a conclusive diagnosis or cause

Physiological findings are consistent with induced illness

Patient does not respond to appropriate treatment

Vitality of patient does not reflect laboratory findings

Signs and symptoms cease when parent/ caregiver does not have access to the child

Only parent/caregiver witnesses onset of symptoms and signs

Unexplained illnesses occur in parent/caregiver and patient's siblings

Parent/caregiver has had exposure to or medical or nursing education on reported illness (eg, a parent with sleep apnea)

Parent/caregiver welcomes painful and intrusive tests

Parent/caregiver becomes anxious if patient improves

Parent/caregiver's lying is confirmed

Medical observations yield findings inconsistent with parent/caregiver reports

Source: Adapted from reference 3

management. At each visit, Ms. S's vital signs, height, and weight are measured. In the therapy sessions, she is taught mindfulness skills as well as CBT. The senior psychiatry resident maintains regular communication with the other clinicians involved in Ms. S's care.

After the first month of treatment, Ms. S undergoes repeat imaging at the gastroenterologist's office that indicates her SMAS is no longer occluded. Ms. S continues to report somatic symptoms, but with mild improvement.

Over the course of approximately 4 months, Ms. $S$ begins to show signs of improvement in her pain, anxiety, and

\section{Clinical Point}

CBT and mindfulnessbased therapy may help relieve symptoms associated with somatic symptom disorder 


\section{Clinical Point}

\section{There may be}

an association

between a parent's

psychological status

and a pediatric

patient's somatic

symptoms

\section{Related Resources}

- Henningsen P. Management of somatic symptom disorder. Dialogues Clin Neurosci. 2018;20(1):23-91.

- Rosic T, Kalra S, Samaan Z. Somatic symptom disorder, a new DSM-5 diagnosis of an old clinical challenge. BMJ Case Rep. 2016: bcr2015212553. doi: 10.1136/bcr-2015-212553.

Drug Brand Name

Doxepin • Silenor

depression. Ms. S begins to feel well enough to get a summer job at a nursing home and expresses enthusiasm when her weight begins to increase. Her mother also became enthused and verbalized her appreciation that her daughter appeared to be improving.

In the fall, Ms. S returns to high school for her senior year but has difficulty getting back into the routine and relating to her old friends. Ms. S continues to perseverate on thoughts of getting sick and her physical symptoms become overwhelming once again. She continues to be focused on any new symptoms she experiences, and to limit the types of foods she eats due to fear of the abdominal pain returning.

After several more months of psychiatric treatment, Ms. S reports significant relief from her abdominal pain, and no longer seeks corrective surgery for her SMAS. Although she occasionally struggles with perseverating thoughts and anxiety about her somatic symptoms such as abdominal pain and worrying about the types of foods she eats and becoming ill, she continues to work through symptoms of her somatic symptom disorder.

\section{The authors' observations}

The main challenge of somatic symptom disorder is the patient's "abnormal illness behavior." ${ }^{2,5,6}$ For pediatric patients, there may an association between a parent's psychological status and the patient's somatic symptoms. Abdominal symptoms in a pediatric patient have a strong association with a parent who presents with depression, anxiety, or somatization. The effects of the parent's psychological status could also manifest in the form of modeling catastrophic thinking or through reinforcement. Parents with certain traits, such as disproportionate worry about pain, may pay more attention to their child's symptoms, and hence, reward the child when he/she reports somatic symptoms. ${ }^{78}$ In the case of Ms. S, her mother did not participate in therapy and the mother's psychiatric history was never obtained.

\section{OUTCOMES Making personal strides}

Ms. $S$ continues to use mindfulness skills as well as CBT to manage her symptoms of somatic symptom disorder. She continues to celebrate her weight gains, denies any thoughts of suicide or self-harm behaviors, and prepares for college by scheduling campus visits and completing admissions applications.

\section{Bottom Line}

Patients with somatic symptom disorder tend to have very high levels of worry about illness. Somatic symptoms in such patients may or may not have a medical explanation. Accurate diagnosis and careful management are necessary to reduce patient distress. Cognitive-behavioral therapy and mindfulness-based therapy may help relieve symptoms associated with this disorder. 


\section{References}

1. Diagnostic and statistical manual of mental disorders, 5th ed. Washington, DC: American Psychiatric Association; 2013.

2. Stern $\mathrm{T}$, Freudenreich $\mathrm{O}$, Smith $\mathrm{F}$, et al. Massachusetts General Hospital Handbook of General Hospital Psychiatry, 7th ed. New York, NY: Elsevier; 2017.

3. Feldman MD, Eisendrath SJ. The spectrum of factitious disorders. Washington, DC: American Psychiatric Association; 1997.

4. Sadock BJ, Sadock VA, Ruiz P. Kaplan \& Sadock's synopsis of psychiatry: behavioral sciences/clinical psychiatry, 11th ed. Philadelphia, PA: Wolters Kluwer; 2014:470.

5. Pilowsky I. The concept of abnormal illness behavior. Psychosomatics. 1990;31(2):207-213.
6. Kirmayer LJ, Looper KJ. Abnormal illness behavior: physiological, psychological and social dimensions of coping with stress. Curr Opin Psychiatry. 2006;19(1): $54-60$.

7. Walker LS, Garber J, Greene JW. Somatic complaints in pediatric patients: a prospective study of the role of negative life events, child social and academic competence, and parental somatic symptoms. J Consult Clin Psychology. 1994;62(6):1213-1221.

8. Van Oudenhove L, Levy RL, Crowell MD, et al. Biopsychosocial aspects of functional gastrointestinal disorders: how central and environmental processes contribute to the development and expression of functional gastrointestinal disorders. Gastroenterology. 2016;150(6):1355-1367.
Clinical Point

Ms. S continues to use mindfulness skills as well as CBT to manage her symptoms of somatic symptom disorder 